\title{
Structure-Driven, Ferroelectric Wake-up Effect for Electrical Fatigue Relief
}

Teng Lu ${ }^{1} \star$, Ye Tian ${ }^{1,2,5}$, Andrew Studer ${ }^{3}$, Qian $\mathrm{Li}^{4}$, Ray L. Withers ${ }^{1}, \mathrm{Li} \mathrm{Jin}^{2}$, Dehong Yu ${ }^{3}$, Zhuo $\mathrm{Xu}^{2}$, Xiaoyong $\mathrm{Wei}^{2, *}$, Yun $\mathrm{Liu}^{1, *}$

1. Research School of Chemistry, The Australian National University, Canberra, Australian Capital Territory 2601, Australia

2. Key Laboratory of the Ministry of Education and International Center for Dielectric Research, School of Electronic Science and Engineering, Xi'an Jiaotong University, Xi'an 710049, China.

3. Australian Centre for Neutron Scattering, Australian Nuclear Science and Technology Organisation, Lucas Heights, New South Wales 2234, Australia

4. State Key Laboratory of New Ceramics and Fine Processing, School of Materials Science and Engineering, Tsinghua University, Beijing 10084, China.

5. School of Materials Science and Engineering, Shaanxi University of Science \& Technology, Xi'an, Shaanxi 710021, China. 


\section{Supporting Information 1: P-E hysteresis loops of AN45LT and AN60LT measured at $1 \mathrm{~Hz}$}
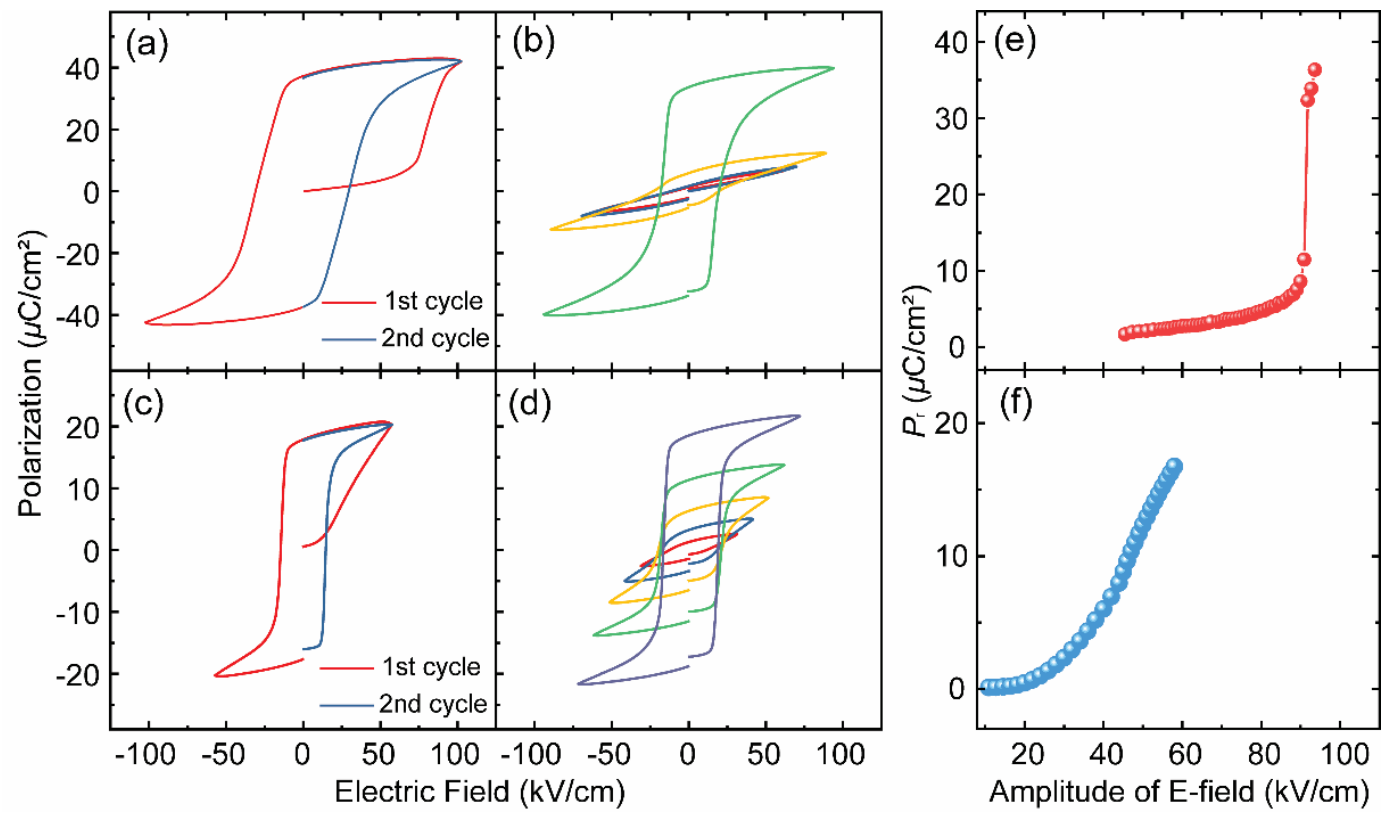

Figure S1. $P-E$ hysteresis loops of AN45LT measured at (a) $1^{\text {st }}$ and $2^{\text {nd }}$ cycles, (b) different applied field amplitudes. P-E hysteresis loops of AN60LT measured at (c) $1^{\text {st }}$ and $2^{\text {nd }}$ cycles, (d) different applied field amplitudes. The remnant polarization $\left(P_{\mathrm{r}}\right)$ as a function of applied field amplitudes for (e) AN45LT and (f) AN60LT. All $P-E$ hysteresis loops are measure at $1 \mathrm{~Hz}$.

The $P$-E hysteresis loops of AN45LT and AN60LT measured at different cycles, and E-field amplitudes are shown in Figure S1. For AN45LT, the sudden increase of the polarization around $90 \mathrm{kV} / \mathrm{cm}$ during the $1^{\text {st }}$ cycle is suggested to be associated with an E-field induced, AFE to FE phase transition. ${ }^{1-2}$ Furthermore, the square shape, single $P-E$ hysteresis loop with $P_{r} \sim 38 \mu \mathrm{C} / \mathrm{cm}^{2}$ measured at the $2^{\text {nd }}$ cycle suggests that the induced FE phase is metastable after withdrawal of the applied field. The $P-E$ hysteresis loops of the AN60LT sample (Figure S1c) measured at different cycles show the similar behavior except that, instead of the sudden jump observed in AN45LT, the polarization increases gradually during the $1^{\text {st }}$ quarter electric cycle. The evolution of the $P$-E hysteresis loops of AN60LT with increasing applied field amplitudes, shown in Figure S1d, is quite different from that in normal FE materials. ${ }^{3-5}$ When the E-field is sufficiently high, around $90 \mathrm{kV} / \mathrm{cm}$ for AN45LT and $60 \mathrm{kV} / \mathrm{cm}$ for AN60LT, the $P-E$ hysteresis loops of both samples follow the typical FE features (coercive field, $E_{\mathrm{C}} \sim$ $25 \mathrm{kV} / \mathrm{cm}$ for AN45LT and $\sim 20 \mathrm{kV}$ for AN60LT, remnant polarization, $P_{\mathrm{r}} \sim 34 \mu \mathrm{C} / \mathrm{cm}^{2}$ for AN45LT and $19 \mu \mathrm{C} / \mathrm{cm}^{2}$ for AN60LT). It is noteworthy that the $P-E$ hysteresis loops of both samples measured at low field, e.g., $70 \mathrm{kV} / \mathrm{cm}$ for AN45LT and $40 \mathrm{kV} / \mathrm{cm}$ for AN60LT (these values surpass $E_{\mathrm{C}}$ shown 
in Figure S1b and d), behave like lossy dielectric materials, whose profiles are quite similar to that of pure $\mathrm{AgNbO}_{3}$ measured at low field. ${ }^{6-7}$ Panels e and fof Figure $\mathrm{S} 1$ plot the $P_{\mathrm{r}}$ of the ANLT samples as a function of the applied field amplitudes. It is found that the relationship of the $P_{\mathrm{r}}$-field amplitudes is quite similar to that of $P-E$ measured during the $1^{\text {st }}$ quarter cycle (Figure S1a and c). This phenomenon can be attributed to the phase transition between the AFE $P m c 2_{1}$ and FE $R 3 c$ phases. The pristine AN45LT sample only contains the $P m c 21$ phase, and a sufficient E-field can change the $P m c 21$ phase into the $R 3 c$ phase, inducing a large $P_{\mathrm{r}}$. For AN60LT, its pristine state contains both $P m c 2_{1}$ and $R 3 c$ phases, suggesting a very flat energy landscape connecting these two phases. With increasing the applied E-field amplitude, both the phase transition and the FE domain reorientation contribute to the $P-E$ hysteresis loop, leading to the gradual increase of $P_{\mathrm{r} .}$ It is noteworthy that although results obtained from Figures S1a, b and e indicate that the sufficient E-field $(>90 \mathrm{kV} / \mathrm{cm})$ could induce the metastable FE phase in the AN45LT sample, the $P-E$ loop measured at the $2^{\text {nd }}$ cycle (blue curve in Figure S1a) shows larger $E_{\mathrm{c}}$ and $P_{\mathrm{r}}$ compared with the green curve shown in Figure $\mathrm{S} 1 \mathrm{~b}$. It is possible because the field applied in Figure $\mathrm{S} 1 \mathrm{a}(100 \mathrm{kV} / \mathrm{cm})$ is quite close to the dielectric breakdown field of the AN45LT sample, and the larger $E_{\mathrm{c}}, P_{\mathrm{r}}$, and slightly tilted $P-E$ loop might result from the leakage current. 
Supporting Information 2: The results of Rietveld refinements of the pristine, intermediate, and woken-up AN45LT and AN60LT samples.
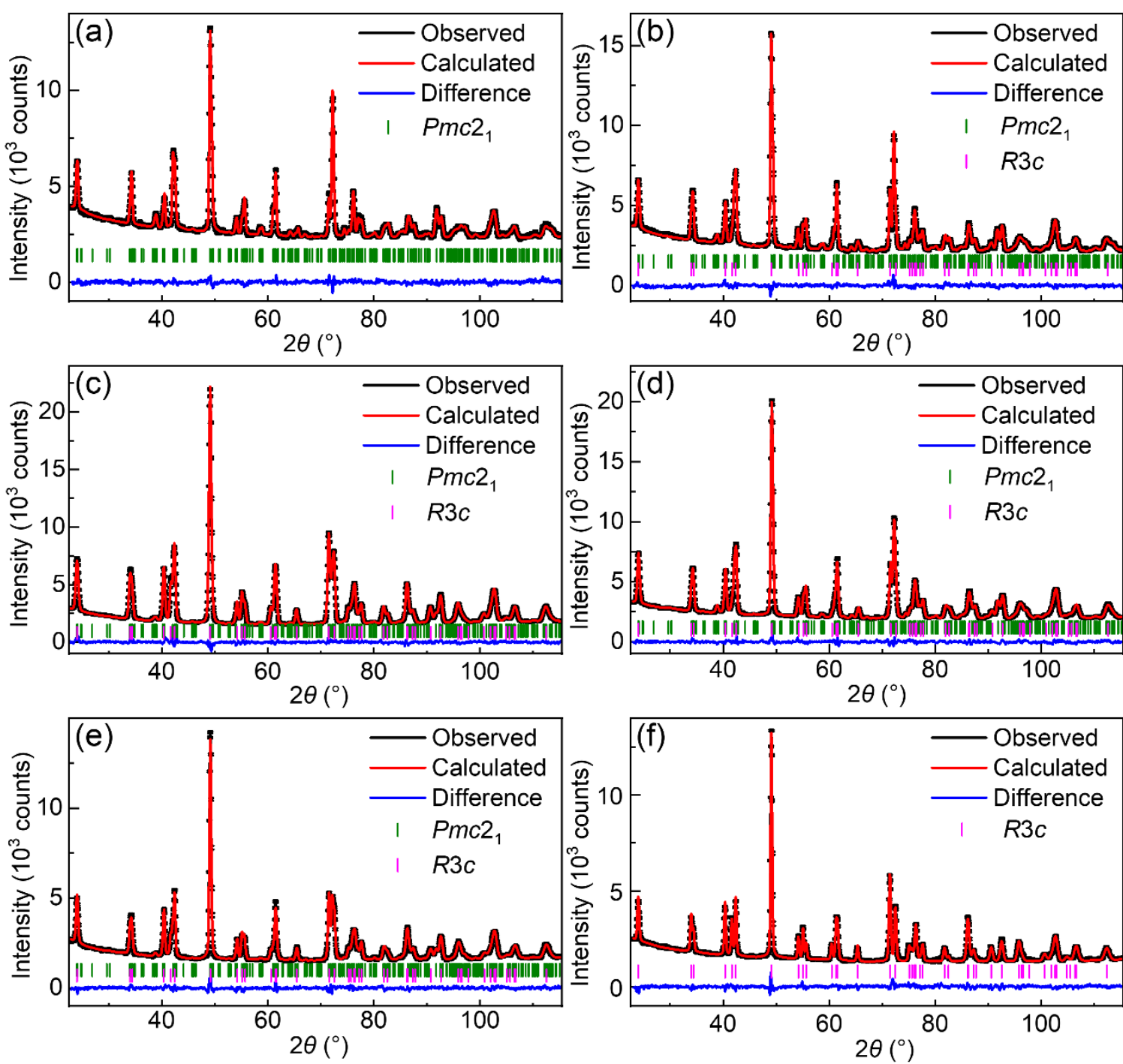

Figure S2. Neutron powder diffraction (NPD) data with the calculated patterns for (a) pristine, (b) IM and (c) woken-up AN45LT and (d) pristine, (e) IM and (f) woken-up AN60LT.

Table S1. The refined crystal lattice parameters and reliability factors in Rietveld analyses of the NPD data for pristine, IM, and woken-up AN45LT and AN60LT.

\begin{tabular}{c|c|ccc|ccccc}
\hline \multirow{2}{*}{ Sample name } & \multirow{2}{*}{$\begin{array}{l}\text { Space } \\
\text { group }\end{array}$} & \multicolumn{4}{|c}{ Unit-cell parameters $(\AA)$} & \multicolumn{4}{c}{ R-factors } \\
\cline { 3 - 10 } & $a$ & $b$ & $c$ & $R_{p}$ & $R w_{p}$ & $R_{e x}$ & $R_{B r a g g}$ & $R_{F}$ \\
\hline Pristine AN45LT & $P m c 21$ & $15.6485(25)$ & $5.5347(12)$ & $5.5965(12)$ & $1.89 \%$ & $2.37 \%$ & $1.76 \%$ & $6.22 \%$ & $5.57 \%$ \\
\hline
\end{tabular}




\begin{tabular}{|c|c|c|c|c|c|c|c|c|}
\hline \multirow{2}{*}{ IM AN45LT } & $P m c 2_{1}$ & $15.6500(25)$ & $5.5403(12)$ & $5.6032(10)$ & $2.04 \% 2.57 \%$ & $1.78 \%$ & $7.25 \%$ & $5.29 \%$ \\
\hline & $R 3 c$ & $5.5281(8)$ & $5.5281(8)$ & $13.7910(18)$ & $2.04 \% \quad 2.57 \%$ & $1.78 \%$ & $5.48 \%$ & $2.62 \%$ \\
\hline \multirow{2}{*}{$\begin{array}{l}\text { Woken-up } \\
\text { AN45LT }\end{array}$} & $P m c 2_{1}$ & $15.6561(24)$ & $5.5427(8)$ & $5.6050(7)$ & $2.38 \% 3.10 \%$ & $1.89 \%$ & $4.71 \%$ & $3.28 \%$ \\
\hline & $R 3 c$ & $5.5291(8)$ & $5.5291(8)$ & $13.7922(18)$ & $2.38 \% \quad 3.10 \%$ & $1.89 \%$ & $2.92 \%$ & $1.39 \%$ \\
\hline \multirow{2}{*}{ Pristine AN60LT } & $P m c 2_{1}$ & $15.6570(22)$ & $5.5298(8)$ & $5.5940(7)$ & $2.34 \% \quad 2.98 \%$ & $1.82 \%$ & $4.08 \%$ & $4.06 \%$ \\
\hline & $R 3 c$ & $5.5244(8)$ & $5.5244(8)$ & $13.7779(18)$ & $2.34 \% \quad 2.98 \%$ & $1.82 \%$ & $2.78 \%$ & $1.83 \%$ \\
\hline \multirow{2}{*}{ IM AN60LT } & $P m c 2_{1}$ & $15.6728(26)$ & $5.5323(8)$ & $5.5970(7)$ & $2.27 \% \quad 2.90 \%$ & $2.07 \%$ & $5.91 \%$ & $5.36 \%$ \\
\hline & $R 3 c$ & $5.5266(8)$ & $5.5266(8)$ & $13.7810(18)$ & $2.27 \% \quad 2.90 \%$ & $2.07 \%$ & $4.11 \%$ & $2.40 \%$ \\
\hline $\begin{array}{l}\text { Woken-up } \\
\text { AN60LT }\end{array}$ & $R 3 c$ & $5.5309(8)$ & $5.5309(8)$ & $13.8039(18)$ & $2.72 \% \quad 3.56 \%$ & $2.20 \%$ & $5.21 \%$ & $3.43 \%$ \\
\hline
\end{tabular}

Table S2. Refined atomic positions of NPD data for pristine AN45LT at room temperature

\begin{tabular}{|c|c|c|c|c|c|}
\hline \multirow{2}{*}{$\begin{array}{l}\text { Space group } \\
\text { Atomic label }\end{array}$} & \multirow{2}{*}{$\begin{array}{c}P m c 2_{1} \\
\text { Wyckoff site }\end{array}$} & \multicolumn{3}{|c|}{ Fractional coordinates } & \multirow[t]{2}{*}{$\operatorname{Biso}\left(\AA^{2}\right)$} \\
\hline & & $x$ & $y$ & $z$ & \\
\hline Ag3/Li3 & $2 \mathrm{a}$ & 0 & $0.732(2)$ & $0.2917(18)$ & $0.71(3)$ \\
\hline Ag1/Li1 & $4 c$ & $0.752(2)$ & $0.752(2)$ & $0.2644(16)$ & $0.71(3)$ \\
\hline $\mathrm{Ag} 2 / \mathrm{Li} 2$ & $2 b$ & 0.5 & $0.758(2)$ & $0.2502(18)$ & $0.71(3)$ \\
\hline $\mathrm{Nb} 2 / \mathrm{Ta} 2$ & $4 c$ & $0.1260(8)$ & $0.2371(11)$ & $0.271(2)$ & $0.30(2)$ \\
\hline $\mathrm{Nb} 1 / \mathrm{Ta} 1$ & $4 c$ & $0.6282(9)$ & $0.7539(11)$ & $0.738(2)$ & $0.30(2)$ \\
\hline O5 & $2 \mathrm{a}$ & 0 & $0.1942(19)$ & $0.231(3)$ & $0.632(18)$ \\
\hline $\mathrm{O} 1$ & $4 c$ & $0.7509(11)$ & $0.6990(19)$ & $0.749(3)$ & $0.632(18)$ \\
\hline $\mathrm{O} 2$ & $2 b$ & 0.5 & $0.8077(19)$ & $0.768(3)$ & $0.632(18)$ \\
\hline O6 & $4 c$ & $0.1413(9)$ & $0.0174(16)$ & $-0.010(2)$ & $0.632(18)$ \\
\hline $\mathrm{O} 7$ & $4 c$ & $0.1144(9)$ & $0.4824(16)$ & $0.530(2)$ & $0.632(18)$ \\
\hline $\mathrm{O} 4$ & $4 \mathrm{c}$ & $0.6418(9)$ & $0.0446(16)$ & $0.562(2)$ & $0.632(18)$ \\
\hline $\mathrm{O} 3$ & $4 c$ & $0.6079(9)$ & $0.5397(16)$ & $0.483(2)$ & $0.632(18)$ \\
\hline
\end{tabular}

Table S3. Refined atomic positions of NPD data for IM AN45LT at room temperature

\begin{tabular}{cccccc}
\hline Space group & Pmc2 1 & \multicolumn{3}{c}{ Fractional coordinates } & \multirow{2}{*}{$\operatorname{Biso}\left(\AA^{2}\right)$} \\
\cline { 1 - 5 } Atomic label & Wyckoff site & $x$ & $y$ & $z$ & \\
\cline { 1 - 5 } $\mathrm{Ag} / \mathrm{Li} 3$ & 2a & 0 & $0.729(2)$ & $0.293(2)$ & $0.75(4)$ \\
$\mathrm{Ag} 1 / \mathrm{Li} 1$ & $4 \mathrm{c}$ & $0.752(2)$ & $0.7512(2)$ & $0.264(1)$ & $0.75(4)$ \\
$\mathrm{Ag} 2 / \mathrm{Li} 2$ & 2b & 0.5 & $0.7618(9)$ & $0.248(2)$ & $0.75(4)$ \\
$\mathrm{Nb} 2 / \mathrm{Ta} 2$ & 4c & $0.1261(3)$ & $0.2519(12)$ & $0.2682(17)$ & $0.43(2)$
\end{tabular}




\begin{tabular}{cccccc} 
Nb1/Ta1 & 4c & $0.6282(3)$ & $0.7393(11)$ & $0.7313(17)$ & $0.43(2)$ \\
O5 & 2a & 0 & $0.1865(11)$ & $0.2200(19)$ & $0.70(2)$ \\
O1 & $4 c$ & $0.751(2)$ & $0.6913(11)$ & $0.7400(19)$ & $0.70(2)$ \\
O2 & 2b & 0.5 & $0.8155(11)$ & $0.7608(19)$ & $0.70(2)$ \\
O6 & $4 c$ & $0.1414(5)$ & $0.013(2)$ & $-0.000(2)$ & $0.70(2)$ \\
O7 & $4 c$ & $0.1202(5)$ & $0.482(2)$ & $0.521(2)$ & $0.70(2)$ \\
O4 & $4 c$ & $0.6463(5)$ & $0.044(2)$ & $0.566(2)$ & $0.70(2)$ \\
O3 & $4 c$ & $0.6093(5)$ & $0.534(2)$ & $0.479(2)$ & $0.70(2)$ \\
\hline Space group & R3c & \multicolumn{5}{c}{ Fractional coordinates } & Biso $\left(\AA^{2}\right)$ \\
\hline Atomic label & Wyckoff site & $x$ & $y$ & $z$ & \\
\hline Ag1/Li1 & 6a & 0 & 0 & 0.2663 & $0.75(4)$ \\
Nb1/Ta1 & 6a & 0 & 0 & $0.0187(8)$ & $0.43(2)$ \\
O1 & $18 b$ & $0.5495(14)$ & $0.9887(19)$ & $0.2581(4)$ & $0.70(2)$ \\
\hline
\end{tabular}

Table S4. Refined atomic positions of NPD data for woken-up AN45LT at room temperature

\begin{tabular}{|c|c|c|c|c|c|}
\hline Space group & $P m c 2_{1}$ & \multicolumn{3}{|c|}{ Fractional coordinates } & \multirow[t]{2}{*}{$\operatorname{Biso}\left(\AA^{2}\right)$} \\
\hline Atomic label & Wyckoff site & $x$ & $y$ & $z$ & \\
\hline Ag3/Li3 & $2 \mathrm{a}$ & 0 & $0.725(1)$ & $0.280(3)$ & $1.19(6)$ \\
\hline Ag1/Li1 & $4 \mathrm{c}$ & $0.752(2)$ & $0.748(1)$ & $0.271(2)$ & $1.19(6)$ \\
\hline $\mathrm{Ag} 2 / \mathrm{Li} 2$ & $2 b$ & 0.5 & $0.773(2)$ & $0.248(3)$ & $1.19(6)$ \\
\hline $\mathrm{Nb} 2 / \mathrm{Ta} 2$ & $4 c$ & $0.1261(3)$ & $0.2310(18)$ & $0.249(3)$ & $0.52(3)$ \\
\hline $\mathrm{Nb} 1 / \mathrm{Ta} 1$ & $4 \mathrm{c}$ & $0.6282(3)$ & $0.7601(18)$ & $0.722(2)$ & $0.52(3)$ \\
\hline O5 & $2 \mathrm{a}$ & 0 & $0.193(2)$ & $0.218(2)$ & $1.02(5)$ \\
\hline $\mathrm{O} 1$ & $4 \mathrm{c}$ & $0.751(2)$ & $0.697(2)$ & $0.744(2)$ & $1.02(5)$ \\
\hline $\mathrm{O} 2$ & $2 b$ & 0.5 & $0.809(2)$ & $0.771(2)$ & $1.02(5)$ \\
\hline O6 & $4 \mathrm{c}$ & $0.1389(9)$ & $0.011(3)$ & $-0.009(2)$ & $1.02(5)$ \\
\hline $\mathrm{O} 7$ & $4 c$ & $0.1168(9)$ & $0.489(3)$ & $0.503(2)$ & $1.02(5)$ \\
\hline $\mathrm{O} 4$ & $4 \mathrm{c}$ & $0.6441(9)$ & $0.040(3)$ & $0.552(2)$ & $1.02(5)$ \\
\hline $\mathrm{O} 3$ & $4 \mathrm{c}$ & $0.6057(9)$ & $0.535(3)$ & $0.466(2)$ & $1.02(5)$ \\
\hline Space group & $R 3 c$ & \multicolumn{3}{|c|}{ Fractional coordinates } & $\operatorname{Biso}\left(\AA^{2}\right)$ \\
\hline Atomic label & Wyckoff site & $x$ & $y$ & $z$ & \\
\hline Ag1/Li1 & $6 a$ & 0 & 0 & 0.2663 & $1.19(6)$ \\
\hline $\mathrm{Nb} 1 / \mathrm{Ta} 1$ & $6 \mathrm{a}$ & 0 & 0 & $0.0204(7)$ & $0.52(3)$ \\
\hline $\mathrm{O} 1$ & $18 \mathrm{~b}$ & $0.5610(6)$ & $1.0056(9)$ & $0.2578(8)$ & $1.02(5)$ \\
\hline
\end{tabular}

Table S5. Refined atomic positions of NPD data for pristine AN60LT at room temperature

\begin{tabular}{ccccc}
\hline Space group & $P m c 21$ & & Fractional coordinates & \multirow{2}{*}{$\operatorname{Biso}\left(\AA^{2}\right)$} \\
\cline { 1 - 4 } Atomic label & Wyckoff site & $x$ & $y$ & $z$ \\
\hline
\end{tabular}




\begin{tabular}{|c|c|c|c|c|c|}
\hline Ag3/Li3 & $2 \mathrm{a}$ & 0 & $0.747(2)$ & $0.3037(17)$ & $0.73(4)$ \\
\hline Ag1/Li1 & $4 c$ & $0.750(2)$ & $0.746(2)$ & $0.253(1)$ & $0.73(4)$ \\
\hline $\mathrm{Ag} 2 / \mathrm{Li} 2$ & $2 b$ & 0.5 & $0.7488(9)$ & $0.2596(17)$ & $0.73(4)$ \\
\hline $\mathrm{Nb} 2 / \mathrm{Ta} 2$ & $4 c$ & $0.1233(3)$ & $0.2491(12)$ & $0.276(1)$ & $0.47(2)$ \\
\hline $\mathrm{Nb} 1 / \mathrm{Ta} 1$ & $4 \mathrm{c}$ & $0.6236(3)$ & $0.7516(19)$ & $0.745(1)$ & $0.47(2)$ \\
\hline O5 & $2 \mathrm{a}$ & 0 & $0.2205(19)$ & $0.278(2)$ & $0.57(2)$ \\
\hline $\mathrm{O} 1$ & $4 c$ & $0.750(2)$ & $0.6862(19)$ & $0.778(2)$ & $0.57(2)$ \\
\hline $\mathrm{O} 2$ & $2 b$ & 0.5 & $0.7983(19)$ & $0.796(2)$ & $0.57(2)$ \\
\hline O6 & $4 c$ & $0.1373(5)$ & $0.014(2)$ & $-0.009(2)$ & $0.57(2)$ \\
\hline $\mathrm{O} 7$ & $4 \mathrm{c}$ & $0.1117(5)$ & $0.479(2)$ & $0.531(2)$ & $0.57(2)$ \\
\hline $\mathrm{O} 4$ & $4 c$ & $0.6390(5)$ & $0.026(2)$ & $0.562(2)$ & $0.57(2)$ \\
\hline $\mathrm{O} 3$ & $4 c$ & $0.6047(5)$ & $0.530(2)$ & $0.482(2)$ & $0.57(2)$ \\
\hline Space group & $R 3 c$ & \multicolumn{3}{|c|}{ Fractional coordinates } & $\operatorname{Biso}\left(\AA^{2}\right)$ \\
\hline Atomic label & Wyckoff site & $x$ & $y$ & $z$ & \\
\hline Ag1/Li1 & $6 a$ & 0 & 0 & 0.2663 & $0.73(4)$ \\
\hline $\mathrm{Nb} 1 / \mathrm{Ta} 1$ & $6 a$ & 0 & 0 & $0.0193(8)$ & $0.47(2)$ \\
\hline $\mathrm{O} 1$ & $18 \mathrm{~b}$ & $0.5687(14)$ & $1.009(2)$ & $0.2583(8)$ & $0.57(2)$ \\
\hline
\end{tabular}

Table S6. Refined atomic positions of NPD data for IM AN60LT at room temperature

\begin{tabular}{|c|c|c|c|c|c|}
\hline Space group & $P m c 2_{1}$ & \multicolumn{3}{|c|}{ Fractional coordinates } & \multirow[t]{2}{*}{$\operatorname{Biso}\left(\AA^{2}\right)$} \\
\hline Atomic label & Wyckoff site & $x$ & $y$ & $z$ & \\
\hline Ag3/Li3 & $2 \mathrm{a}$ & 0 & $0.748(2)$ & $0.301(2)$ & $0.62(4)$ \\
\hline Ag1/Li1 & $4 \mathrm{c}$ & $0.750(2)$ & $0.743(2)$ & $0.253(1)$ & $0.62(4)$ \\
\hline Ag2/Li2 & $2 b$ & 0.5 & $0.7535(9)$ & $0.262(1)$ & $0.62(4)$ \\
\hline $\mathrm{Nb} 2 / \mathrm{Ta} 2$ & $4 \mathrm{c}$ & $0.1233(3)$ & $0.2491(12)$ & $0.256(2)$ & $0.25(2)$ \\
\hline $\mathrm{Nb} 1 / \mathrm{Ta} 1$ & $4 \mathrm{c}$ & $0.6236(3)$ & $0.7516(19)$ & $0.737(2)$ & $0.25(2)$ \\
\hline $\mathrm{O} 5$ & $2 \mathrm{a}$ & 0 & $0.2396(19)$ & $0.233(3)$ & $0.48(2)$ \\
\hline $\mathrm{O} 1$ & $4 \mathrm{c}$ & $0.750(2)$ & $0.6900(19)$ & $0.734(3)$ & $0.48(2)$ \\
\hline $\mathrm{O} 2$ & $2 b$ & 0.5 & $0.8010(19)$ & $0.753(3)$ & $0.48(2)$ \\
\hline O6 & $4 c$ & $0.1358(5)$ & $0.041(16)$ & $-0.024(9)$ & $0.48(2)$ \\
\hline $\mathrm{O} 7$ & $4 \mathrm{c}$ & $0.1132(5)$ & $0.4943(16)$ & $0.505(2)$ & $0.48(2)$ \\
\hline $\mathrm{O} 4$ & $4 \mathrm{c}$ & $0.6409(5)$ & $0.030(2)$ & $0.548(2)$ & $0.48(2)$ \\
\hline $\mathrm{O} 3$ & $4 \mathrm{c}$ & $0.6027(5)$ & $0.528(2)$ & $0.474(2)$ & $0.48(2)$ \\
\hline Space group & $R 3 c$ & \multicolumn{3}{|c|}{ Fractional coordinates } & $\operatorname{Biso}\left(\AA^{2}\right)$ \\
\hline Atomic label & Wyckoff site & $x$ & $y$ & $z$ & \\
\hline Ag1/Li1 & $6 a$ & 0 & 0 & 0.2663 & $0.62(4)$ \\
\hline $\mathrm{Nb} 1 / \mathrm{Ta} 1$ & $6 a$ & 0 & 0 & $0.0195(8)$ & $0.25(2)$ \\
\hline $\mathrm{O} 1$ & $18 \mathrm{~b}$ & $0.5626(14)$ & $1.004(2)$ & $0.2566(8)$ & $0.48(2)$ \\
\hline
\end{tabular}

Table S7. Refined atomic positions of NPD data for woken-up AN60LT at room temperature 


\begin{tabular}{cccccc}
\hline Space group & $R 3 c$ & \multicolumn{3}{c}{ Fractional coordinates } & \multirow{2}{*}{$\operatorname{Biso}\left(\AA^{2}\right)$} \\
\cline { 1 - 5 } Atomic label & Wyckoff site & $x$ & $y$ & $z$ & \\
\cline { 1 - 5 } Ag1/Li1 & $6 \mathrm{a}$ & 0 & 0 & 0.2663 & $1.10(4)$ \\
Nb1/Ta1 & $6 \mathrm{a}$ & 0 & 0 & $0.0211(6)$ & $0.55(2)$ \\
O1 & $18 \mathrm{~b}$ & $0.5614(14)$ & $1.0057(9)$ & $0.2567(5)$ & $0.35(2)$ \\
\hline
\end{tabular}

\section{Supporting Information 3: Electron diffraction patterns of ANLT samples:}
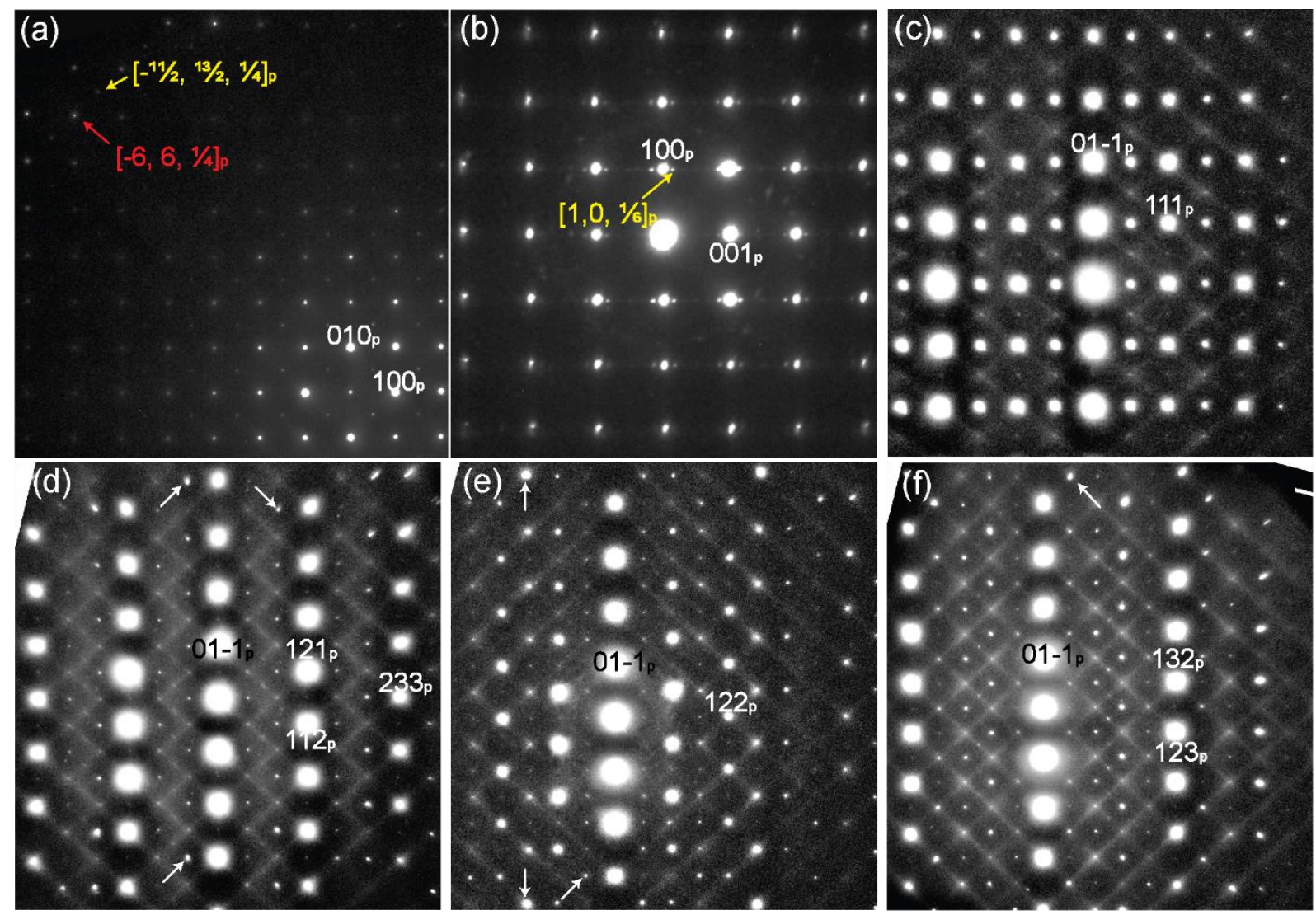

Figure S3. The (a) $<001>_{\mathrm{p}}$, (b) $<010>_{\mathrm{p}}$, zone axis electron diffraction patterns (EDPs) of AN45LT samples. The (c) $<2-1-1>_{p},(d)<3-1-1>_{p},($ e $)<4-1-1>_{p}$ and (f) $<5-1-1>_{p}$ a successive tilt series of zone axis EDPs of AN60LT samples.

Figure S3 above shows the electron diffraction patterns (EDPs) of ANLT samples collected from different zone-axes. All EDPs are indexed referring to the parent perovskite structure (subscript $p$ ). In Figure S3a, in addition to the well-defined set of parent perovskite Bragg reflections $\mathbf{G}_{\mathrm{p}}$, the presence of $\mathbf{G}_{\mathrm{p}} \pm[1 / 2,1 / 2,0]_{\mathrm{p}} *$ reflections with the weak intensities at the zero-order Laue zone (ZOLZ) is consistent with the structure of the $P m c 2_{1}$ phase. Additionally, at the first-order Laue zone (FOLZ), the weak but clear spots, which can be indexed in terms of the $\mathbf{G}_{\mathrm{p}} \pm[1 / 2,1 / 2,1 / 4]_{\mathrm{p}} *$ (arrowed in yellow) and $\mathbf{G}_{\mathrm{p}} \pm[0,0,1 / 4]_{\mathrm{p}} *$ (arrowed in red) further confirm the refined $P m c 2_{1}$ structure obtained from NPD. Figure $3 b$ in the main text shows $\mathbf{G}_{\mathrm{p}} \pm[1 / 2,1 / 2,1 / 3]_{\mathrm{p}} *$ reflections, corresponding to the distorted structure 
condensed at the $T_{4}(1 / 2,1 / 2,1 / 3)$ point. This structure is associated with a $\sqrt{2} a_{\mathrm{p}} \times \sqrt{2} a_{\mathrm{p}} \times 6 a_{\mathrm{p}}$ superlattice which has previously been reported in $\mathrm{NaNbO}_{3}$ and $\mathrm{AgNbO}_{3}$-based perovskite systems. ${ }^{8-10}$ The proposed structure contains a complex octahedral tilting system along the $<001>_{\mathrm{p}}$ direction. For this distorted $6 a_{\mathrm{p}}$ perovskite structure, the modulation wave-vector of the AFE mode correspondingly changes from the $[0,0,1 / 4]_{\mathrm{p}} *$ to the $[0,0,1 / 6]_{\mathrm{p}}$, which have also been found in AN45LT sample (yellow arrowed reflections in Figure S3b).

Figures S3c-f show a series of systematically tilting EDPs of the AN60LT sample. The evident $\mathbf{G}_{\mathrm{p}} \pm[1 / 2$, $1 / 2,1 / 2]_{\mathrm{p}} *$ reflections (Figure $\mathrm{S} 3 \mathrm{c}$ ) are suggested to originate from the antiphase octahedral tilting, which is written as $a^{-} a^{-} c^{0}$ for $P m c 21$ phase and $a^{-} a^{-} a^{-}$for $R 3 c$ phase in Glazer notation. ${ }^{11}$ Intriguingly, with systematically tilting towards $<5-1-1>_{\mathrm{p}}$ zone axis, a series of satellite reflections, $\mathbf{G}_{\mathrm{p}} \pm[1 / \mathrm{n}, 1 / 2,1 / 2]_{\mathrm{p}} *$ (where $\mathrm{n}$ is 3, 4 and 5), is present. These satellite reflections have strong intensities at the boundary of the EDPs while becoming weaker at the center (denoted by the white arrows), which indicates that these apparent 'reflections' are in fact cuts through rods of diffuse scattering, $\mathbf{G}_{\mathrm{p}} \pm[1 / 2,1 / 2, \xi]_{\mathrm{p}} *(\xi$ continuous). Such a diffuse streaking denotes disordered octahedral tilting around the $<\begin{array}{llll}0 & 0 & 1>_{p}\end{array}$ direction. Besides the satellite reflections, the $\mathbf{G}_{\mathrm{p}} \pm<h k 0>_{\mathrm{p}} *$ diffuse streaking running through the Bragg reflections of parent perovskite structure, $\mathbf{G}_{\mathrm{p}}$, is obtained at these $\langle u v w\rangle$ orientations (Figures $3 \mathrm{c}-\mathrm{f})$, e.g., the diffuse streaking along the $<-120>_{\mathrm{p}} *$ direction in Figure S3c. The characteristic diffuse streaking is always perpendicular to $<0 \quad 01>_{p}$ real space direction, which indicates the correlation of atomic displacements along the $<001>_{p}$ direction. ${ }^{12-13}$

\section{Supporting Information 4: PFM results of the pristine AN60LT sample}
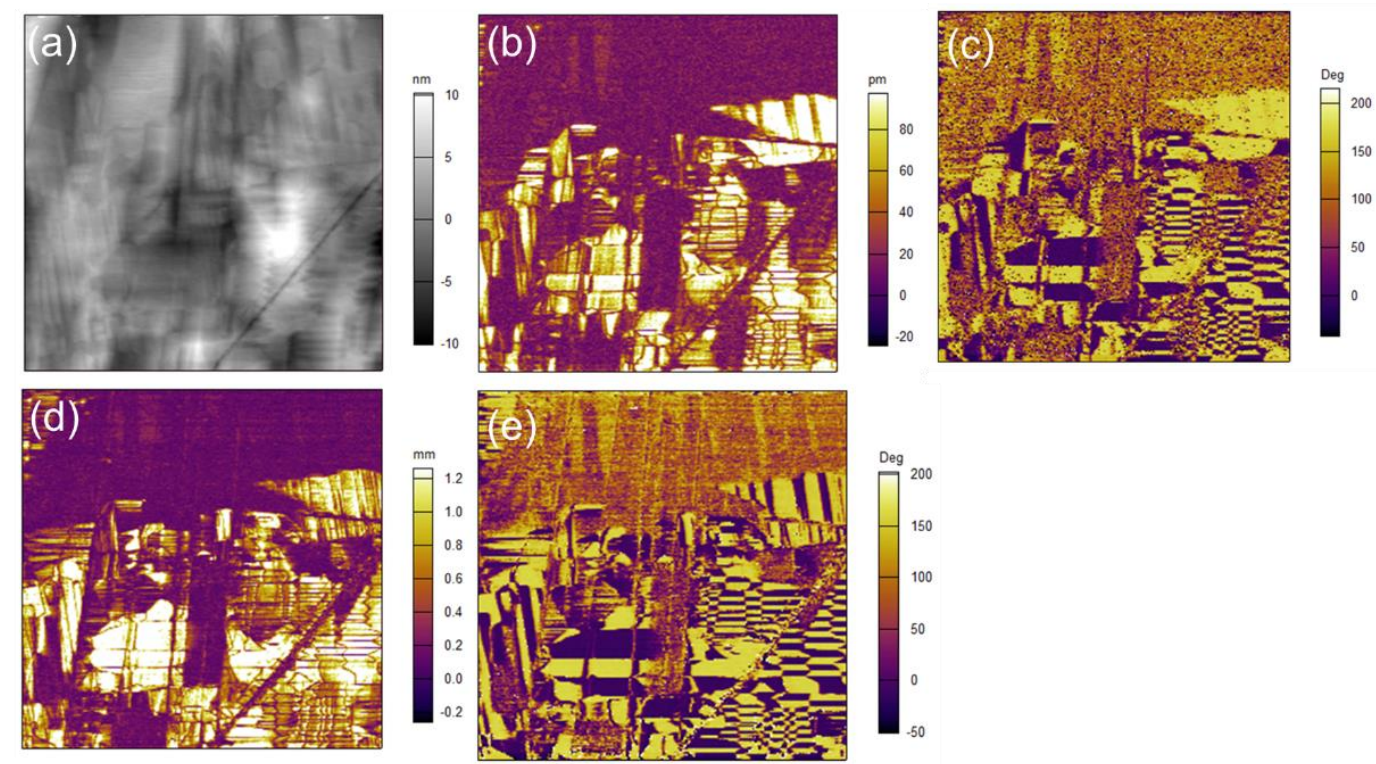

Figure S4. (a) Morphology, (b) vertical amplitude, (c) vertical phase, (d) lateral amplitude and (e) 
lateral phase of $10 \times 10 \mu \mathrm{m}$ area on the pristine AN60LT pellet.

Supporting Information 5: The wake-up processes of AN60LT ceramics under different bipolar cycling fields.
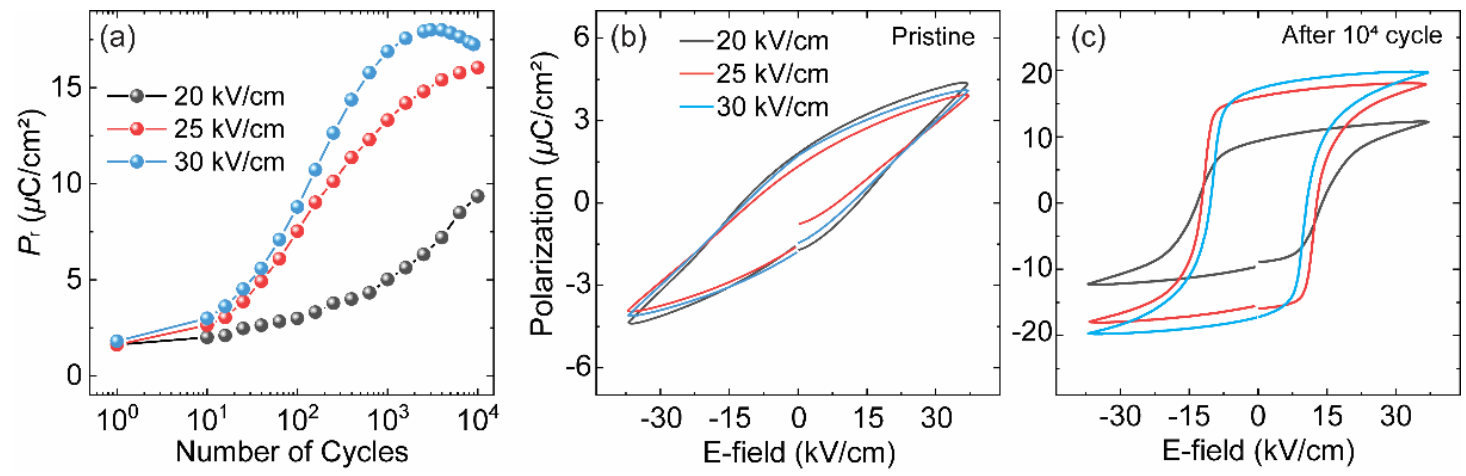

Figure S5. (a) Evolution of the remnant polarization of AN60LT during the different bipolar cycling. The $P-E$ hysteresis loops of AN60LT measured (b) before and (c) after $10^{4}$ bipolar cyclic E-field with different amplitudes. The frequency for all cycling fields is $10 \mathrm{~Hz}$, and all $P$-E hysteresis loops are measure at $1 \mathrm{~Hz}$.

Supporting Information 6: $P-E$ hysteresis loops of BNBT and BNBT-ANLT samples measured before and after $10^{4}$ bipolar field cycles
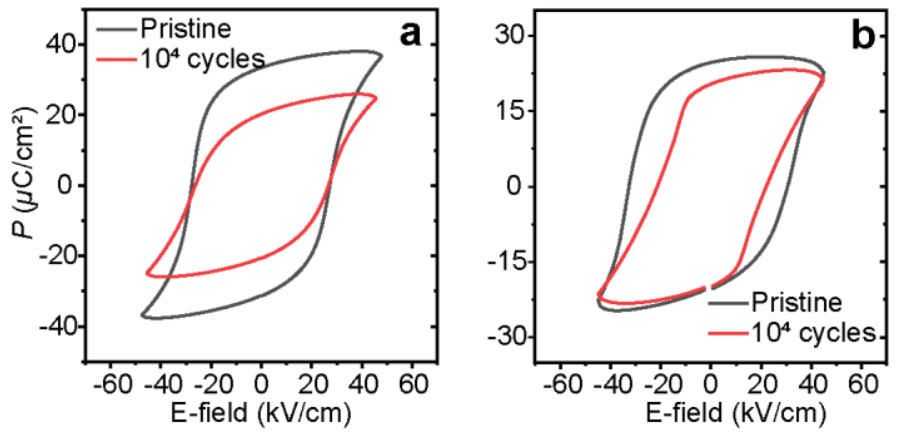

Figure S6. Fatigue effects on $P-E$ hysteresis loops of the a) BNBT and b) BNBT-AN60LT samples. 


\section{References:}

1.Berlincourt, D.; Krueger, H. H. A.; Jaffe, B., Stability of Phases in Modified Lead Zirconate with Variation in Pressure, Electric Field, Temperature and Composition. J. Phys. Chem. Solids 1964, 25 (7), 659-674.

2.Tan, X.; Frederick, J.; Ma, C.; Jo, W.; Rödel, J., Can an Electric Field Induce an Antiferroelectric Phase Out of a Ferroelectric Phase? Phys. Rev. Lett. 2010, 105 (25), 255702.

3.Jin, L.; Li, F.; Zhang, S., Decoding the Fingerprint of Ferroelectric Loops: Comprehension of the Material Properties and Structures. J. Am. Ceram. Soc. 2014, 97 (1), 1-27.

4.Zhou, D.; Guan, Y.; Vopson, M. M.; Xu, J.; Liang, H.; Cao, F.; Dong, X.; Mueller, J.; Schenk, T.; Schroeder, U., Electric Field and Temperature Scaling of Polarization Reversal in Silicon Doped Hafnium Oxide Ferroelectric Thin Films. Acta Mater. 2015, 99, 240-246.

5.Peng, P.; Nie, H.; Liu, Z.; Wang, G.; Dong, X.; Zhang, Y.; Duan, C.; Tang, X., Scaling Behavior for $\left(\mathrm{Bi}_{0.5} \mathrm{Na}_{0.5}\right) \mathrm{TiO}_{3}$ Based Lead-Free Relaxor Ferroelectric Ceramics. J. Appl. Phys. 2017, 122 (6), 064102.

6.Fu, D.; Endo, M.; Taniguchi, H.; Taniyama, T.; Itoh, M., AgNbO3: A Lead-Free Material with Large Polarization and Electromechanical Response. Appl. Phys. Lett. 2007, 90 (25), 3.

7.Tian, Y.; Jin, L.; Zhang, H.; Xu, Z.; Wei, X.; Politova, E. D.; Stefanovich, S. Y.; Tarakina, N. V.; Abrahams, I.; Yan, H., High Energy Density in Silver Niobate Ceramics. J. Mater. Chem. A 2016, 4 (44), 17279-17287.

8.Mishra, S. K.; Mittal, R.; Pomjakushin, V. Y.; Chaplot, S. L., Phase Stability and Structural Temperature Dependence in Sodium Niobate: A High-Resolution Powder Neutron Diffraction Study. Phys. Rev. B 2011, 83 (13).

9.Peel, M. D.; Thompson, S. P.; Daoud-Aladine, A.; Ashbrook, S. E.; Lightfoot, P., New Twists on the Perovskite Theme: Crystal Structures of the Elusive Phases $\mathrm{R}$ and $\mathrm{S}$ of $\mathrm{NaNbO}_{3}$. Inorg. Chem. 2012, 51 (12), 6876-6889. 10.Khan, H. U.; Sterianou, I.; Miao, S.; Pokorny, J.; Reaney, I. M., The Effect of Li-Substitution on the MPhases of $\mathrm{AgNbO}_{3}$. J. Appl. Phys. 2012, 111 (2), 024107.

11.Glazer, A. M., Simple Ways of Determining Perovskite Structures. Acta Crystallogr., Sect. A: Cryst. Phys., Diffr., Theor. Gen. Crystallogr. 1975, 31 (6), 756-762.

12.Liu, Y.; Withers, R. L.; Wei, X.; Fitz Gerald, J. D., Structured Diffuse Scattering and Polar Nano-Regions in the $\mathrm{Ba}\left(\mathrm{Ti}_{1-x} \mathrm{Sn}_{x}\right) \mathrm{O}_{3}$ Relaxor Ferroelectric System. J. Solid State Chem. 2007, 180 (3), 858-865.

13.Levin, I.; Krayzman, V.; Woicik, J. C.; Karapetrova, J.; Proffen, T.; Tucker, M. G.; Reaney, I. M., Structural Changes Underlying the Diffuse Dielectric Response in $\mathrm{AgNbO}_{3}$. Phys. Rev. B 2009, 79 (10), 104113. 